\title{
Progression model tissue microarray (TMA) for the study of uterine carcinomas
}

\author{
Mohammad Arafa ${ }^{\mathrm{a}}$, Jacques Boniver ${ }^{\mathrm{b}}$ and Philippe Delvenne $\mathrm{b}^{\mathrm{b}, *}$ \\ ${ }^{a}$ Department of Pathology, Faculty of Medicine, Mansoura University, Mansoura, Egypt \\ ${ }^{\mathrm{b}}$ Department of Anatomic Pathology and Cytology, GIGA-Cancer, University of Liege, Liege, Belgium
}

\begin{abstract}
Cervical and endometrial uterine carcinomas are heterogeneous groups of cancers, which are preceded by preneoplastic lesions. More accurate tools are needed to improve the diagnosis and to define markers which may be relevant for the diagnosis, prediction of disease progression and therapeutic response.

High throughput technologies for testing and validating molecular targets in cancer lesions and in their precursors are presently available. Among them, the tissue microarray (TMA) presents the advantage of a morphological control of the analyzed tissue fragment. In this article, we review the different aspects of the TMA technology with a special consideration to a uterine carcinogenesis model.
\end{abstract}

\section{Introduction}

The uterus is divided anatomically into cervix and corpus. The epithelial lining of the uterine cervix is squamous in the ectocervical part and glandular in the endocervix with a transformation zone between the two regions. The uterine corpus lining, known as the endometrium, is of glandular epithelial nature. In broad terms, primary malignant epithelial neoplasms of the cervix may be either squamous cell carcinoma or, less commonly, adenocarcinoma [32]. On the other hand, carcinoma of the endometrium is usually an adenocarcinoma [27]. Both carcinomas (cervical and endometrial) have different etiologic factors mainly in the form of HPV infection (viral carcinogenesis) for the former and unopposed estrogen (hormonal carcinogenesis) for the later [8,12]. Moreover, both carcinomas (cervical and endometrial) evolve through recognizable and treatable pre-invasive stages referred to as cervical intraepithelial neoplasia (CIN) and adenocarcinoma in situ (AIS) for cervical squamous and glandular lesions, respectively [32]. Similarly, endometrial adenocarci-

* Corresponding author: Philippe Delvenne, MD, PhD, Department of Pathology B35, Chu Sart Tilman, 4000 Liege, Belgium. Tel.: +32 43662564; Fax: +32 43662919; E-mail: p.delvenne@ulg.ac.be. noma follows a preneoplastic stage called atypical hyperplasia $(\mathrm{AH})$ which represents a major fraction of endometrial intraepithelial neoplasia (EIN), the precursor lesion of Type I endometrial carcinoma [16]. Early detection and accurate diagnosis of these neoplasms, especially in the preinvasive stage, reduces their impact as health problems and improves the patients' clinical management and outcome. For this purpose, large scale studies are needed to identify novel genes or target proteins to be selected for diagnostic and therapeutic needs. It is always challenging to identify, validate and select the best targets from thousands of candidate genes and proteins. High throughput technologies for molecular target validation are therefore essential to develop new medical and biotechnological applications. This has resulted in the development of a number of methods to systematically analyze candidate molecular function. One powerful technology that has emerged recently is tissue microarray (TMA) [19].

\section{The TMA technology}

Conventional histopathological studies are usually performed on slides with one section from a single specimen. Therefore, the screening for new anti- 


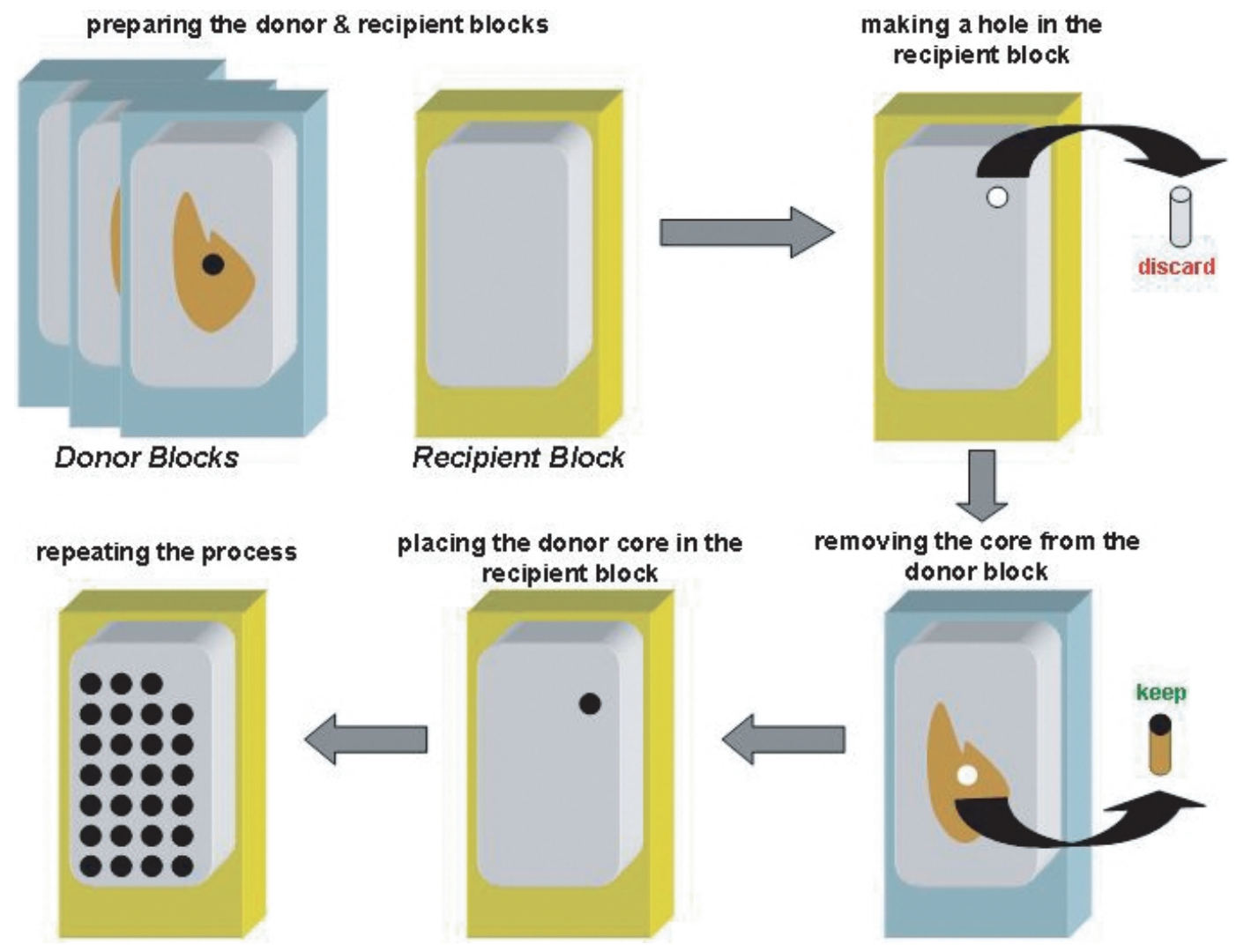

Fig. 1. The process of arraying. Carefully selected areas from the donor blocks are removed and inserted in a defined manner within the recipient block.

bodies of potential clinical value is costly and timeconsuming [29]. To overcome this problem, Battifora developed a method by which multiple normal or tumour tissues can be mounted on a single slide, the multi-tissue or "sausage" block technique in 1986 [7]. Afterwards, they further improved the multi-tissue block technique in 1990 [6]. This technique permits the tissues to be evenly distributed in a checkerboard arrangement and to be readily identified by their position in the resulting sections. Toward this end, one powerful technology that has emerged in 1998 is the tissue microarray (TMA), a technology that permits the high throughput in situ analysis of specific molecular targets in hundreds or thousands of tissue specimens at once [19]. TMAs are collections of arrayed tissue spots on a microscope glass slide that provide a template for highly parallel localization of molecular targets [29].

\section{Construction of TMA}

TMAs are constructed by acquiring cylindrical core from morphologically representative areas of individ- ual tissue samples (donor blocks), followed by the insertion of the cores into a new "recipient" TMA block at defined array locations (Fig. 1). Selection of tissue specimens and the exact histological area to be sampled are the first steps in the TMA construction. Using a special device, the tissue arrayer, cores from up to 1000 tissue samples are arrayed into a recipient TMA block [19,29].

\section{Applications of TMA}

TMA has so far mainly been used in cancer research. Typical tumour TMAs that have been constructed include multi-tumour and cancer progression or prognostic arrays [29].

Multi-tumour TMA may be composed of samples from multiple tumour types. These arrays are usually utilized to screen different tumour types for molecular alterations of interest [1].

TMA has also been used to study molecular alterations in different stages of progression in one type of 
tumour. For example, TMAs have been constructed, including samples representing all stages of prostate cancer development, starting from normal prostate, benign prostate hyperplasia, prostatic intraepithelial neoplasia, localized clinical cancer, to metastatic and homonerefractory end-stage cancer $[9,24]$.

TMA can also be constructed with samples from tumours with clinical follow-up data and clinical endpoints. With the help of such arrays, novel prognostic parameters can be identified and the value of molecular alterations for prediction of chemotherapy response can be tested [21,28].

Although most of the applications of the TMA technique have been described in cancer research, it is likely that the technology will be equally powerful in other fields of research, such as those related to inflammatory, cardiovascular or neurological diseases [5].

\section{Advantages of TMA}

Compared with traditional large section studies, TMA has many advantages. The TMA block can be cut into approximately 200 sections of 3-5 $\mu \mathrm{m}$ thickness, depending on the care and skill of the histotechnologist, to provide slides with the identical configuration of the tissue spots (rows and columns) in each section [29]. Therefore, each section can be used to study a molecular marker and hence a high number of different markers can be tested in hundreds or even thousands samples [19].

The second advantage is that each tissue sample on a TMA slide is treated in an identical manner. This uniformity helps in assuring the specificity and sensitivity of immunohistochemistry (IHC) and improving reproducibility of the staining reaction as well as the speed and reliability of the interpretation [29].

The third advantage is its high speed. An automated tissue arrayer has been introduced [18]. It enhances the speed of molecular analyses to increase by more than 100-fold. Compared with the TMA technique, conventional techniques for molecular analysis are labor intensive and time-consuming [21].

The fourth advantage is that only a small amount of expensive reagents is required to analyze an entire array in a single experiment. This advantage raises the possibility of using TMA in screening procedures [29].

Finally, TMA markedly reduces the amount of archival tissue required for a particular study thus preserving sufficient remaining tissue for other research or diagnostic needs [30].

\section{Limitations of TMA}

The major potential limitation of this technique is the tissue volume, raising the questions of how can a very small core $(0.6 \mathrm{~mm})$ be representative of the whole tumour specimen (because of tumour heterogeneity) and how many cores would be needed to provide the same level of information as an entire section? To address the influence of tumour heterogeneity and to evaluate the ability of TMA to yield information on the prognostic value of biomarkers, some studies have directly compared biomarker expression using TMA and regular sections of the same breast cancers [29]. All studies report more than $90 \%$ concordance for common breast cancer biomarkers such as the estrogen receptor (ER), the progesterone receptor (PR), and the HER-2 oncoprotein [10,11,13,17,29]. These studies showed that analysis of TMA generates results which are representative of the entire tumour, and then appropriate for analysing the prognostic significance of such markers $[10,11,13,17,29]$. One difficulty with paraffinembedded tissues relates to antigenic changes in proteins and mRNA degradation induced by the fixation and embedding process. To overcome such limitations, this technology has been modified by using frozen tissues embedded in OCT compound as "donor" samples and arraying the specimens into a "recipient" OCT block [26].

\section{Progression model TMA for uterine carcinomas}

Study of the multistep pathogenesis of uterine carcinomas and the discovery of target molecules for diagnostic and therapeutic implications requires the analysis of a large series of hysterectomy specimens. In order to provide a continuum of control tissues, normal tissues from diseased women, preneoplastic lesions and infiltrative carcinomas, TMA models have been developed for cervical and endometrial carcinogenesis (Fig. 2) [2,3].

Several technical issues should be considered for TMA construction and interpretation. As this process requires careful selection, mapping and arraying of the areas of interest, a corresponding H\&E stained slide should be obtained from each donor block and used as a guide to assess morphology and to select an area that represents the specimen [20,29]. Surface epithelial structures (normal, metaplasic and dysplastic epithelium) are more often incorrectly arrayed than infiltrative carcinomas. Consequently, the process should 

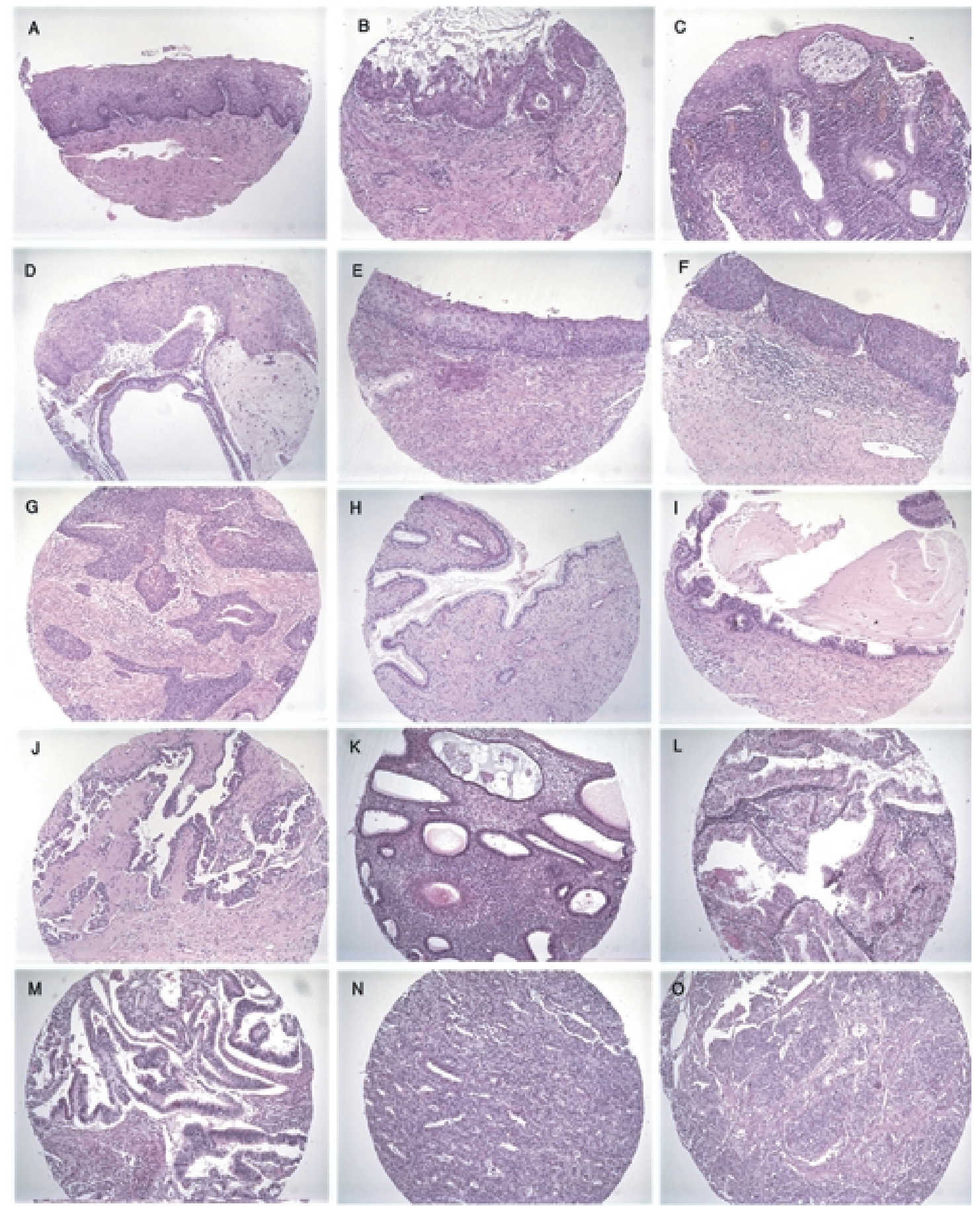

Fig. 2. Different diagnostic entities used for the construction of the tissue microarray (TMA). The uterine cervical squamous carcinogenesis model as constructed by normal ectocervix (A), immature $(\mathbf{B})$ and mature $(\mathbf{C})$ squamous metaplasia, cervical intraepithelial neoplasia (CIN) grades I (D), II (E) and III (F) as well as squamous cell carcinoma (G).The uterine cervical glandluar carcinogenesis model as constructed by normal endocervix $(\mathbf{H})$, adenocarcinoma insitu $(\mathbf{I})$ and primary cervical adenocarcinoma $(\mathbf{J})$. The endometrial carcinogenesis model as constructed by normal endometrium $(\mathbf{K})$, atypical endometrial hyperplasia $(\mathbf{L})$ and endometrioid carcinoma of the endometrium grades I $(\mathbf{M})$, II $(\mathbf{N})$, III $(\mathbf{O})$. (H\&E x 100). 


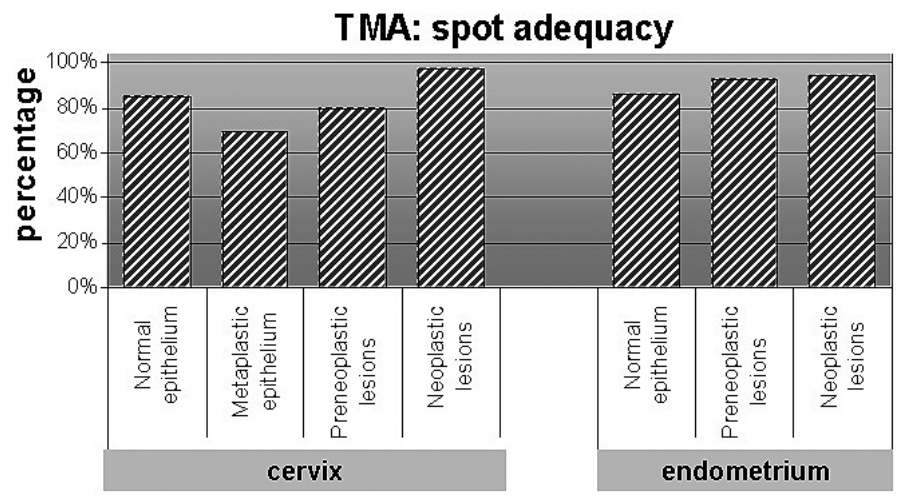

Fig. 3. Adequate tissue specimens as percentages of the totally arrayed tissue microarray (TMA) spots.

be more precise aiming at providing enough epithelium and including parts of the subepithelium as well [25].

The appropriate choice of the diameter of the tissue spots is also of importance for the adequacy of the arrayed tissue spots. In our experience and that of other investigators, arraying small focal areas in a complex tissue such as surface epithelium, squamous metaplasia, CIN and AIS was satisfactory with cores having relatively large diameter $(1 \mathrm{~mm})[2,4,20]$. Despite the fact that cores of large diameter result in the reduction of the total number of the arrayed tissue spots, the use of such "low-density" array facilitates screening of the slides and reduces the likelihood of losing tissue spots during the process of cutting the TMA block [14]. However, the tension inside the recipient block is increased and this could be avoided by constructing special large paraffin recipient blocks [20].

To get as many high-quality sections from one multitissue block as possible, it is advisable to array uniformly the tissue cores at the same level [15]. It is also recommended to array long tissue cores of no less than $2 \mathrm{~mm}$ thickness, although this is not possible in all cases $[15,29]$. In our work we found some donor blocks to be thinner (less than $1.5 \mathrm{~mm}$ ) and, therefore, punching of long cores was not possible. To overcome this problem, more than one short core from the same tumour area can be punched and stacked on top of another in the same recipient location on the tissue array [29].

Concerning the undetectable tissue spots (incorrectly arrayed or detached during cutting/staining procedures), we found, in concordance with previous works, that spot adequacy of more than $80 \%$ could be achieved with the use of single tissue cores [15,31]. However, the majority of inadequate spots, mainly incorrectly arrayed, in our TMAs were observed for lesions of focal, fluctuating or dynamic nature such as CIN or metaplasia. By contrast, owing to precise mapping and arraying, infiltrative carcinomas having wider surface areas than preneoplastic lesions were adequately presented in about $95 \%$ in their corresponding TMA spots (Fig. 3) [25]. It is proved that the use of one tissue core per lesion has a chance of $20 \%$ inadequately arrayed spots. However, this problem is of a minor concern if the focus of the study is the expression profile of tumour population rather than in-depth individual case analysis [14,15,31].

As any technique, TMA has its limitations. The most obvious drawback that has been extensively discussed in the literature is the reliability of TMA to reflect tissue heterogeneity compared to whole tissue sections $[4,10$, $11,13,17,29]$. With consideration of the tumour type and the nature of target molecule, we agree that data obtained from a single tissue core per specimen is sufficient to drive most of the information about the expression profiles of a given cellular population [2,14,31]. For example, ovarian carcinoma which is notorious of its extreme heterogeneity showed that the analysis of a single readable core matches the staining pattern of a whole section in more than $90 \%$ of the cases [23]. However, it was reported that a better mirror image could be obtained with the arraying of multiple tissue cores per specimen [30,31]. Moreover, it is recommended that using large $(>6 \mathrm{~mm})$ core diameters could overcome the problem of tissue heterogeneity [4]. This proposal was opposed by other investigators who appreciate large core diameter to guard against sampling error rather than tissue heterogeneity [30].

To avoid antigenic loss from the remaining unused TMA slides due to tissue oxidation, we and other investigators recommend coating the slides with paraffin prior to storage [22,29].

In conclusion, TMA is a powerful tool for high throughput analysis of molecular targets. Progression models built with the different stages of disease (pre- 
neoplastic stage to invasive tumour) are less common and more difficult to construct. However, they yield valuable information about molecular characteristics of the disease and provide precious material for future studies.

\section{References}

[1] C.L. Andersen, O.M. Monni, J. Kononen et al., High-throughput gene copy number analysis in 4700 tumors: FISH analysis on tissue microarrays identifies multiple tumor types with amplification of the MB-174 gene, a novel amplified gene originally found in breast cancer, Am J Hum Genet 67 (2000), 448.

[2] M. Arafa, J. Boniver and P. Delvenne. Detection of HPVinduced cervical (pre) neoplastic lesions: a tissue microarray (TMA) study, Appl Immunohistochem Mol Morphol 16 (2008), 422-432.

[3] M. Arafa, F. Kridelka, V. Mathias et al., High frequency of RASSF1A and RARb2 gene promoter methylation in morphologically normal endometrium adjacent to endometrioid adenocarcinoma, Histopathology 53 (2008), 525-532.

[4] S. Avninder, K. Ylaya and S.M. Hewitt, Tissue microarray: A simple technology that has revolutionized research in pathology, J Postgrad Med 54 (2008), 158-162.

[5] L.W. Ayers, Application of the tissue microarray (TMA) method by the midregion AIDS and Cancer Specimen Bank (ACSB) to prepare study sets from HIV infected and control tissues, J Acquir Immune Defic Syndr 23 (2000), A18.

[6] H. Battifora, The multitumor (sausage) tissue block: novel method for immunohistochemical antibody testing, Lab Invest 55 (1986), 244-248

[7] H. Battifora and P. Mehta. The checkerboard tissue block. An improved multitissue control block, Lab Invest 63 (1990), $722-724$.

[8] F.X. Bosch, M.M. Manos, N. Muñoz et al., Prevalence of human papillomavirus in cervical cancer: a worldwide perspective. International biological study on cervical cancer (IBSCC) Study Group, J Natl Cancer Inst 87 (1995), 796-802.

[9] C. Bowen, L. Bubendorf, H.J. Voeller et al., Loss of NKX3.1 expression in human prostate cancers correlates with tumor progression, Cancer Res 60 (2000), 6111-6115.

[10] C. Bucher, J. Torhorst, L. Bubendorf et al., Tissue microarrays ('tissue chips') for high-throughput cancer genetics:Linking molecular changes to clinical endpoints, Am J Hum Genet 65(suppl) (1999), 43.

[11] R.L. Camp, L.A. Charette and D.L. Rimm, Validation of tissue microarray technology in breast carcinoma, Lab Invest $\mathbf{8 0}$ (2000), 1943-1949.

[12] G. Emons, G. Fleckenstein, B. Hinney et al., Hormonal interactions in endometrial cancer, Endocr Relat Cancer 7 (2000), 227-242.

[13] C.E. Gillett, R.J. Springall, D.M. Barnes et al., Multiple tissue core arrays in histopathology research: a validation study, $J$ Pathol 192 (2000), 549-553.

[14] W. Gomaa, Y. Ke, H. Fujii et al., Tissue microarray of head and neck squamous carcinoma: validation of the methodology for the study of cutaneous fatty acid-binding protein, vascular endothelial growth factor, involucrin and Ki-67, Virchows Arch 447 (2005), 701-709.
[15] C. Gulmann and A. O'Grady, Tissue microarrays: an overview, Current Diagnostic Pathology 9 (2003), 149-154.

[16] J.L. Hecht and G.L. Mutter, Molecular and pathologic aspects of endometrial carcinogenesis, J Clin Oncol 24 (2006), 47834791.

[17] A. Hoos, M.J. Urist, A. Stojadinovic et al., Validation of tissue microarrays for immunohistochemical profiling of cancer specimens using the example of human fibroblastic tumors, Am J Pathol 158 (2001), 1245-1251.

[18] O.P. Kallioniemi, U. Wagner, J. Kononen et al., Tissue microarray technology for high-throughput molecular profiling of cancer, Hum Mol Genet 10 (2001), 657-662.

[19] J. Kononen, L. Bubendorf, A. Kallioniemi et al., Tissue microarrays for high-throughput molecular profiling of tumor specimens, Nat Med 4 (1998), 844-847.

[20] J. Packeisen, E. Korsching, H. Herbst et al., Demystified... tissue microarray technology, Mol Pathol 56 (2003), 198-204.

[21] J. Richter, U. Wagner, J. Kononen et al., High-throughput tissue microarray analysis of cyclin E gene amplification and overexpression in urinary bladder cancer, Am J Pathol 157 (2000), 787-794.

[22] D.L. Rimm, R.L. Camp, L.A. Charette et al., Amplification of tissue by construction of tissue microarrays, Exp Mol Pathol 70 (2001), 255-264.

[23] D.G. Rosen, X. Huang, M.T. Deavers et al., Validation of tissue microarray technology in ovarian carcinoma, Mod Pathol 17 (2004), 790-797.

[24] M.A. Rubin, R. Dunn, M. Strawderman et al., Tissue microarray sampling strategy for prostate cancer biomarker analysis, Am J Surg Pathol 26 (2002), 312-319.

[25] V. Schneider, Tissue microarrays for testing molecular biomarkers of cervical intraepithelial neoplasia: feasibility study, Acta Cytol 50 (2006), 123-128.

[26] M. Schoenberg Fejzo and D.J. Slamon, Frozen tumor tissue microarray technology for analysis of tumor RNA, DNA, and proteins, Am J Pathol 159 (2001), 1645-1650.

[27] S.G. Silverberg, G.L. Mutter, R.J. Kurman et al., Tumors of the uterine corpus: epithelial tumors and related lesions, in: Pathology and Genetics of Tumours of the Breast and Female Genital Organs, F.A. Tavassoli and P. Devilee, eds, World Health Organization Classifocation of Tumours. IARC Press, Lyon, 2003, pp. 221-232.

[28] R. Simon, J. Richter, U. Wagner et al., High-throughput tissue microarray analysis of 3p25 (RAF1) and 8p12 (FGFR1) copy number alterations in urinary bladder cancer, Cancer Res $\mathbf{6 1}$ (2001), 4514-4519.

[29] Z. Tang and Y. Lu, High-throughput tissue microarray technology for the rapid clinical translation of genomic discoveries, in: Frontiers in Biochip Technology, W.L. Xing and J. Cheng, eds, Springer, 2006, pp. 324-343.

[30] M. Tawfik El-Mansi and A.R. Williams, Validation of tissue microarray technology using cervical adenocarcinoma and its precursors as a model system, Int J Gynecol Cancer 16 (2006), 1225-1233.

[31] A. Tzankov, P. Went, A. Zimpfer et al., Tissue microarray technology: principles, pitfalls and perspectives - lessons learned from hematological malignancies, Exp Gerontol 40 (2005), 737-744.

[32] M. Wells, A.G. Östör, C.P. Crum et al., Epithelial tumors of the uterine cervix, in: Pathology and Genetics of Tumours of the Breast and Female Genital Organs, F.A. Tavassoli and P. Devilee, eds, World Health Organization Classifocation of Tumours. IARC Press, Lyon, 2003, pp. 221-232. 


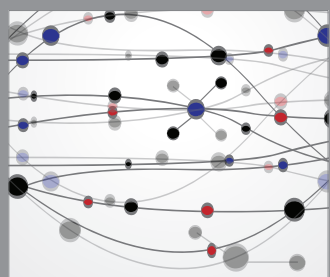

The Scientific World Journal
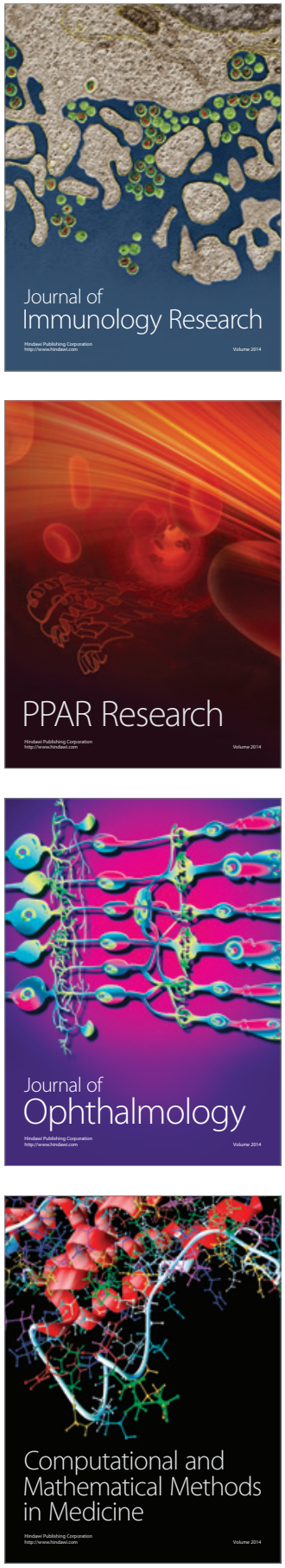

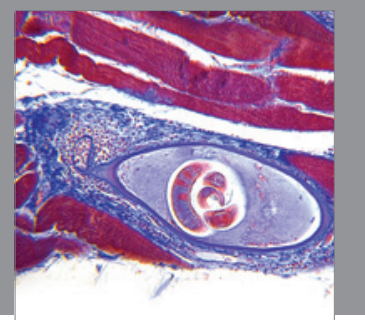

Gastroenterology

Research and Practice
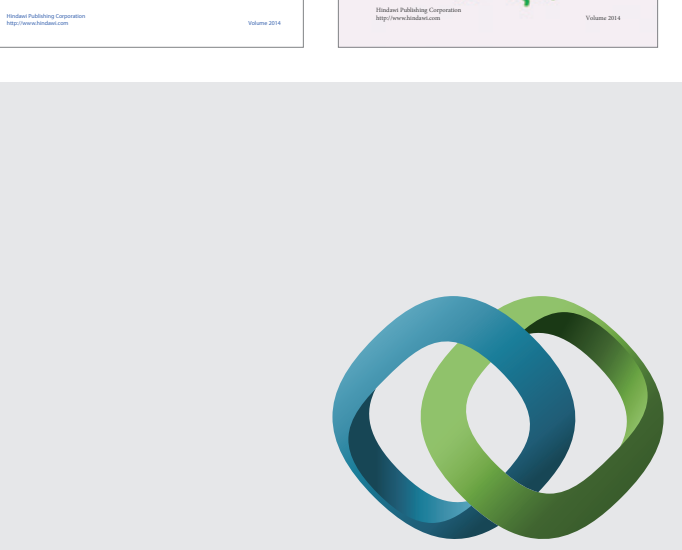

\section{Hindawi}

Submit your manuscripts at

http://www.hindawi.com
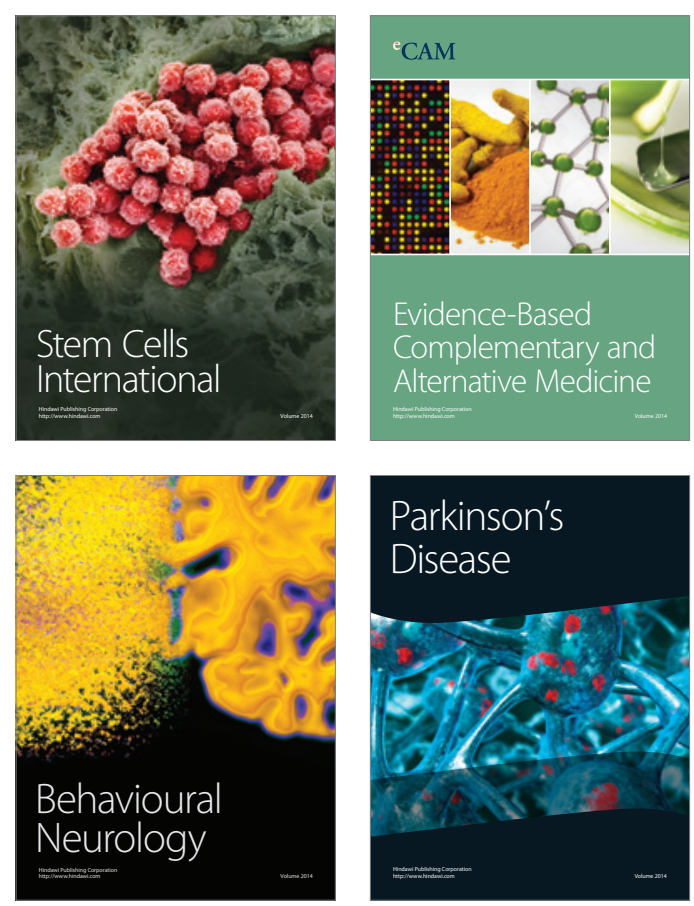

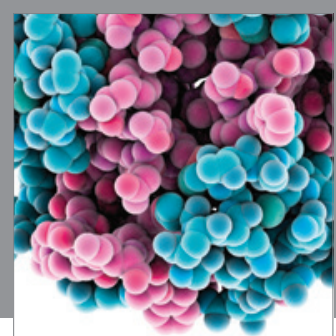

Journal of
Diabetes Research

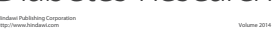

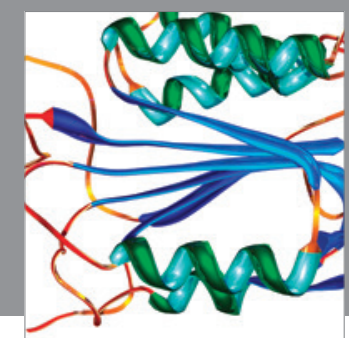

Disease Markers
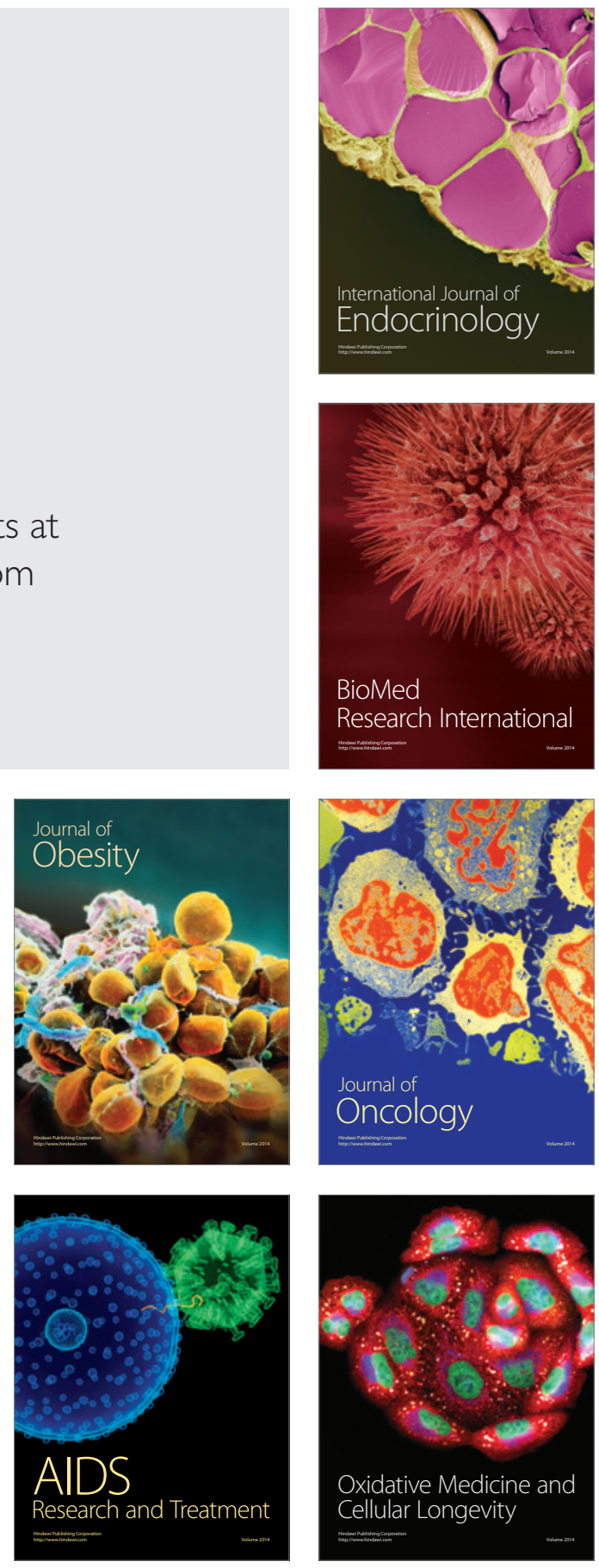